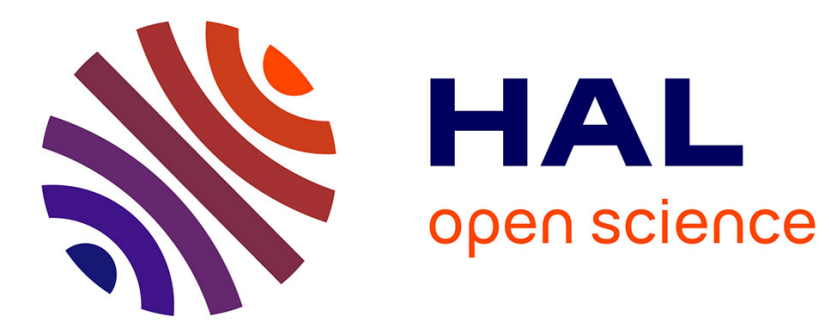

\title{
Aero-acoustics source separation with sparsity inducing priors in the frequency domain
}

Olivier Schwander, José Picheral, Nicolas Gac, Ali-Mohammad Djafari, Daniel Blacodon

\section{- To cite this version:}

Olivier Schwander, José Picheral, Nicolas Gac, Ali-Mohammad Djafari, Daniel Blacodon. Aeroacoustics source separation with sparsity inducing priors in the frequency domain. 34th International Workshop on Bayesian Inference and Maximun Entropy Methods in Science and Engineering (MaxEnt'14), Sep 2014, Amboise, France. pp.422 - 431, 10.1063/1.4906006 . hal-01103779

\section{HAL Id: hal-01103779 \\ https://hal-centralesupelec.archives-ouvertes.fr/hal-01103779}

Submitted on 15 Jan 2015

HAL is a multi-disciplinary open access archive for the deposit and dissemination of scientific research documents, whether they are published or not. The documents may come from teaching and research institutions in France or abroad, or from public or private research centers.
L'archive ouverte pluridisciplinaire HAL, est destinée au dépôt et à la diffusion de documents scientifiques de niveau recherche, publiés ou non, émanant des établissements d'enseignement et de recherche français ou étrangers, des laboratoires publics ou privés. 


\title{
Aero-acoustics source separation with sparsity inducing priors in the frequency domain
}

\author{
Olivier Schwander*,, José Picheral*, Nicolas Gac ${ }^{\dagger}$, Ali-Mohammad \\ Djafari $^{\dagger}$ and Daniel Blacodon** \\ * Département Signal et Systèmes Électroniques (SSE), SUPELEC, Gif-sur-Yvette, France \\ ${ }^{\dagger}$ Laboratoire des signaux et systèmes (L2S), UMR 8506 CNRS-SUPÉLEC-Univ. \\ Paris-Sud, \\ SUPÉLEC, Plateau de Moulon, 91192 Gif-sur-Yvette, France \\ ** Onera, Chatillon, France
}

\begin{abstract}
The characterization of acoustic sources is of great interest in many industrial applications, in particular for the aeronautic or automotive industry for the development of new products. While localization of sources using observations from a wind tunnel is a well-known subject, the characterization and separation of the sources still needs to be explored. We present here a Bayesian approach for sources separation. Two prior modeling of the sources are considered: a sparsity inducing prior in the frequency domain and an autoregressive model in the time domain. The proposed methods are evaluated on synthetic data simulating noise sources emitting from an airfoil inside a wind tunnel.
\end{abstract}

Keywords: Bayesian Source Separation, Joint Maximum A Posteriori (JMAP), Sparsity, Auto-regressive prior, Windtunnel.

\section{INTRODUCTION}

The characterization of acoustic sources is of great interest in numerous aeroacoustic applications. In particular, the prediction of positions and absolute power levels is an important challenge for the aeronautic industry during the development of a new aircraft, mainly to reduce annoying noises around airports and in urban areas. The problem of discovering the location of noise sources and estimating the power levels has been efficiently solved using deconvolution based methods $[1,2]$ but the characterization and separation of the noise sources is still an open problem. This characterization in the time domain is very important as it would allow a better understanding of the physical processes generating the noise.

Previous studies on airfoils using acoustic imaging techniques have shown that the main source of interest is localized on the trailing edge of the airfoil. We use here experimental data collected inside the anechoic wind tunnel Cepra 19 on a NACA12 profile ( FIGURE 1). Using the prior knowledge on the position of the noise source of interest, the airfoil has been equipped with pressure sensors on the trailing edge: due to their position, these sensors are sensitive both to the noise generated locally by the narrow band sources and by the air flow noise inside the tunnel. The objective of this study is to separate the noise emitted from the trailing edge of the airfoil from the other noise sources which are not of interest to us. 


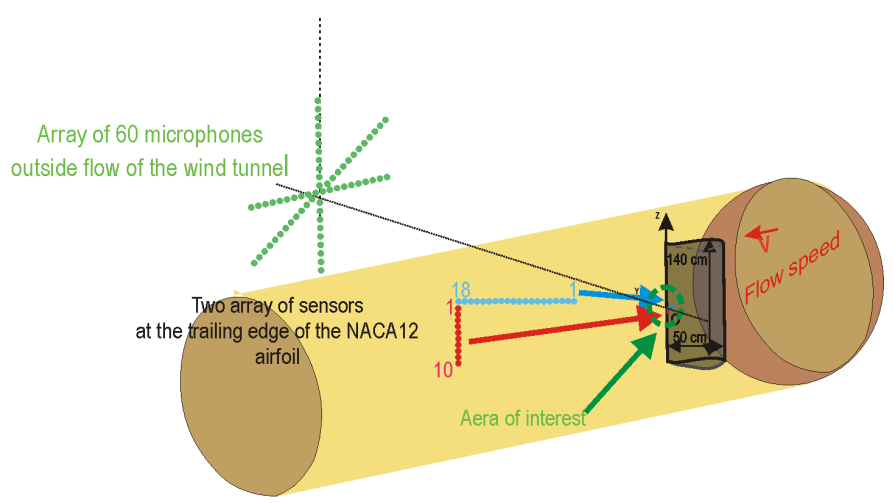

FIGURE 1. Position of airfoil pressure sensors inside the wind tunnel

Difficulties arise since the power level of the not interesting aero-acoustic noises is much higher than the power level from the trailing edge source (40dB between the two sources). Fortunately there is some physical prior knowledge available to ease the separation: the interest source is supposed to be stationary and to have a sparse and unimodal frequency distribution. In order to exploit this prior knowledge, we use a classical Bayesian approach for source separation. Thus, the physical prior on the distribution of frequencies is translated into a sparsity inducing prior. The first possible approach is to work directly in the frequency domain, using a heavytail prior distribution such as the Laplace distribution or the generalized Gaussian distribution. The other choice is to put an indirect constraint on the spectrum with an auto-regressive prior in the temporal domain. Parameters of this auto-regressive model can be chosen (or learned as hyper-parameters) in order to impose a narrow band. In both cases, the solution of the Maximum A Posteriori (MAP) problem is numerically computed using a joint maximization method.

This article is organized as follows: the first section describes the physical setup along with its model; the second section presents the Bayesian methods and algorithms used; the last section validates our proposed methods on a simulated dataset.

\section{PHYSICAL SETUP}

\section{Wind tunnel}

A schematic view of the studied airfoil and of the microphones is presented in FIGURE 2. The airfoil is instrumented with two sets of microphones: a first one is longitudinal to the air flow (at the middle of the airfoil) and the second one is transverse, along the trailing edge. In both cases, the sensors are separated by $4.5 \mathrm{~mm}$. The very low distance between sensors, compared to the wavelength of the studied signal (between 0 and $5 \mathrm{kHz}$ ), allows us to ignore propagation delays.

The spectrum of two observations from real data is shown in FIGURE 3: a first sample comes from a microphone outside the airflow and a second one comes 


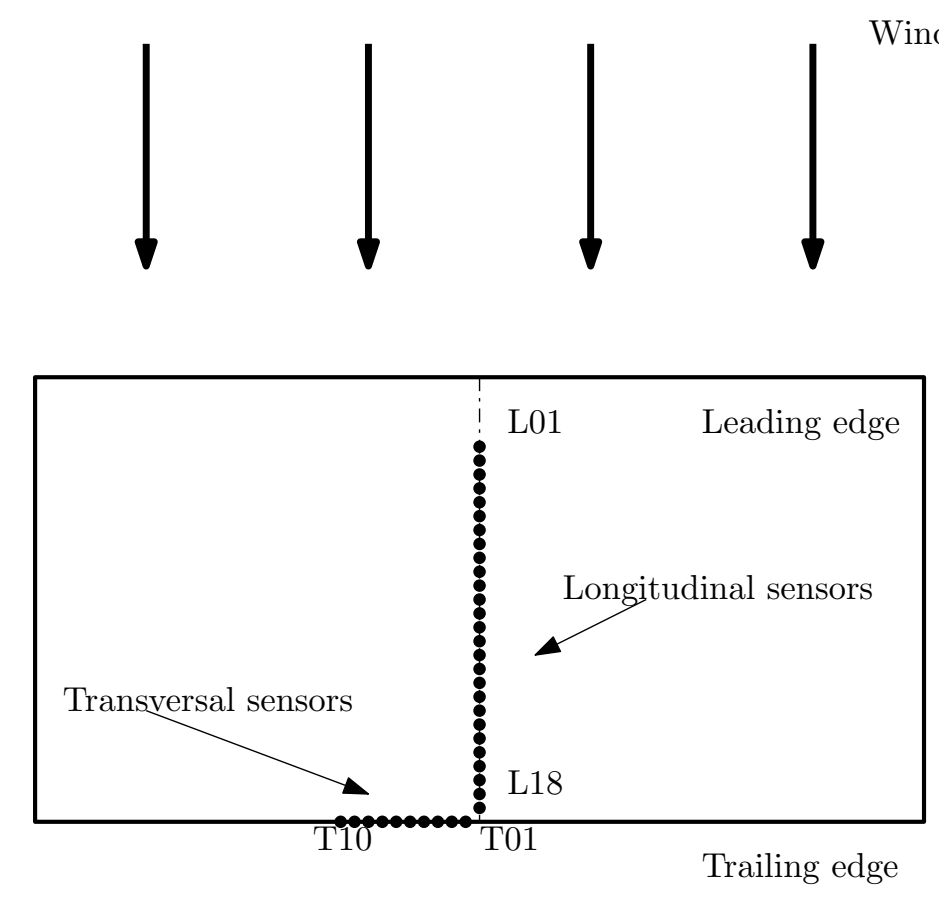

FIGURE 2. Details of the sensor position on the airfoil

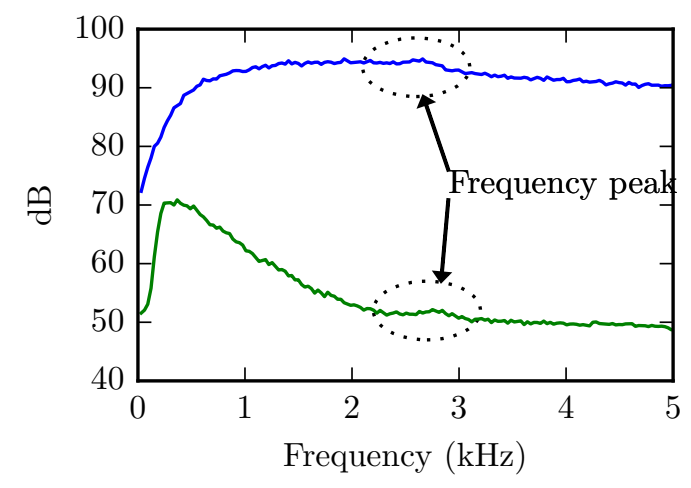

FIGURE 3. Acoustic noise received by microphones on the airfoil (top) and outside the airflow (bottom). In both cases the peak from the narrow band source is visible.

from a microphone on the airfoil. In both cases, the narrow band interesting signal is clearly visible around $2800 \mathrm{~Hz}$. We can also notice that the microphone inside the airflow hear the acoustic noise (peak 0 and $1000 \mathrm{~Hz}$ ) from the wind inside the tunnel, contrary to the sensor outside the flow. 


\section{Forward model}

The acoustic signal received by the microphone $m$ at time $t$ is the mixture of the large band source $s_{1}$ and the narrow band source $s_{2}$ :

$$
x_{m}(t)=a_{m 1} s_{1}(t)+a_{m 2} s_{2}(t)+e_{m}(t) \text { for } m \in[1 \ldots M]
$$

where $e_{m}(t)$ represents the modeling and measurement errors and $a_{m 1}, a_{m 2}$ are the mixing coefficients.

Putting $x_{m}(t)$ for $m \in[1 \ldots M]$ and $t \in[1 \ldots T]$ in the matrix $\mathbf{X}$, the mixing coefficient $a_{m 1}$ (resp. $a_{m 2}$ ) in the column vector $\mathbf{A}_{1}$ (resp. $\mathbf{A}_{2}$ ), the source samples $s_{1}(t)$ (resp. $s_{2}(t)$ ) in the row vector $\mathbf{S}_{1}$ (resp. $\mathbf{S}_{2}$ ) and the error terms $e_{m}(t)$ in the matrix $\mathbf{E}$, the forward model can be written in matrix form as:

$$
\mathbf{X}=\mathbf{A}_{1} \mathbf{S}_{1}+\mathbf{A}_{2} \mathbf{S}_{2}+E
$$

We can also write the mixing relation in the frequencies space:

$$
\mathbf{x}_{m}(\nu)=a_{m 1} \mathbf{s}_{1}(\nu)+a_{m 2} \mathbf{s}_{2}(\nu)+\mathrm{e}_{m}(\nu)
$$

which can be also written in matrix form as:

$$
\mathbf{X}=\mathbf{A}_{1} \mathbf{S}_{1}+\mathbf{A}_{2} \mathbf{S}_{2}+\mathbf{E}
$$

The forward model will be used in one version or another depending on the prior knowledge modeling: with the sparsity inducing prior the frequential version must be used and with the $\operatorname{AR}(2)$ model, the temporal version is needed.

In both cases, the error term $\mathbf{E}$ is supposed to be a white zero-mean Gaussian distribution.

\section{BAYESIAN SOURCE SEPARATION}

\section{Posterior probability}

Using the previously described forward model, we can write the posterior probability as:

$$
p\left(\mathbf{A}, \mathbf{S}_{1}, \mathbf{S}_{2} \mid \mathbf{X}, \theta\right) \propto p\left(\mathbf{X} \mid \mathbf{A}, \mathbf{S}_{1}, \mathbf{S}_{2}, \theta_{E}\right) p\left(\mathbf{A} \mid \theta_{A}\right) p\left(\mathbf{S}_{1} \mid \theta_{1}\right) p\left(\mathbf{S}_{2} \mid \theta_{2}\right)
$$

where

- $\theta=\left(\theta_{E}, \theta_{A}, \theta_{1}, \theta_{2}\right)$ is the vector of hyper-parameters, 
- $p\left(\mathbf{A} \mid \theta_{A}\right)$ is the prior probability for the mixing matrix,

- $p\left(\mathbf{S}_{1} \mid \theta_{1}\right)$ is the prior probability for the source $\mathbf{S}_{1}$,

- and $p\left(\mathbf{S}_{2} \mid \theta_{2}\right)$ is the prior probability for the source $\mathbf{S}_{2}$.

In order to focus on the modeling of the prior and to simplify the algorithms, we assume in a first step that the hyper-parameters are known or fixed a priori. A further refinement of the method would be to assign prior probabilities to the hyper-parameters in order to estimate them automatically (for all parameters or only a subset of them).

Assigning a zero-mean separable Gaussian probability law to the error term $\mathbf{E}$ and using the forward model, we get:

$$
p\left(\mathbf{X} \mid \mathbf{A}, \mathbf{S}, \theta_{E}\right) \propto \exp \left(-\frac{1}{2 \sigma_{E}^{2}}\|\mathbf{X}-\mathbf{A} \mathbf{S}\|_{2}^{2}\right)
$$

We also assign a zero-mean separable Gaussian probability law to the elements of the mixing matrix $\mathbf{A}$ :

$$
p\left(\mathbf{A}, \theta_{A}\right) \propto \exp \left(-\frac{1}{2 \sigma_{A}^{2}}\|\mathbf{A}\|_{2}^{2}\right)
$$

For the source $S_{1}$, we also use a zero-mean Gaussian distribution:

$$
p\left(\mathbf{S}_{1}, \theta_{\mathbf{S}_{1}}\right) \propto \exp \left(-\frac{1}{2 \sigma_{\mathbf{S}_{1}}^{2}}\left\|\mathbf{S}_{1}\right\|_{2}^{2}\right)
$$

The priors for the sparse source $\mathbf{S}_{2}$ are discussed in the next section.

\section{Sparsity inducing priors}

In order to model the prior knowledge about the sparsity of the trailing edge source, we can either work in the frequency domain, by directly imposing a sparsity constraint on the distribution of frequencies, or work in the time domain, using an indirect constraint to enforce the band limitedness of the frequencies.

\section{Laplace prior}

For the sparsity constraint in the frequency domain, we will use a heavy-tailed distribution such as the Laplace distribution. We thus have the following prior for the $S_{2}$ source:

$$
p\left(\mathbf{S}_{2}\right) \propto \exp \left(-\alpha_{2}\left\|\mathbf{S}_{2}\right\|_{1}\right)
$$


where $\|\cdot\|_{1}$ denotes the $L_{1}$ norm: $\|\mathbf{M}\|_{1}=\sum_{i j}\left|m_{i j}\right|$. The use of the $L_{1}$ norm will impose the sparsity of the signal [3] in the frequency domain but may not produce a narrow band signal (since there is no constraint on the position of the null coefficients). We will see in the experimental part that this is still sufficient to produce a unique frequency peak on our simulated data.

\section{Auto-regressive prior}

Another choice is to remain in the time domain by using an auto-regressive model of order $2(\mathrm{AR}(2))$ for the source $\mathbf{S}_{2}$, which imposes a linear relationship between the signal at time $t$ and its previous values:

$$
S_{2}(t)=\phi_{1} S_{2}(t-1)+\phi_{2} S_{2}(t-2)+e(t)
$$

where $S_{2}(t)$ denotes the coefficient of index $t$ in the vector $\mathbf{S}_{2}$ and $e(t)$ is the innovation at each time $t$ and follows a centered Gaussian law:

$$
p\left(e(t), \theta_{A}\right)=\propto \exp \left(-\frac{1}{2 \sigma_{e}^{2}}\|e(t)\|_{2}^{2}\right)
$$

Using the two hyper-parameters $\phi_{1}$ and $\phi_{2}$ we can control the shape of the spectrum of the source $S_{2}$, since the spectrum of an auto-regressive signal is given by the transfer function $H$ of the associated auto-regressive filter:

$$
H(\omega)=\frac{1}{\left|1-\phi_{1} * e^{-i * \omega}-\phi_{2} * e^{-2 i * \omega}\right|^{2}}
$$

The probability of the sources at time $t$ (for $t>2$ ) given sources at time $t-1$ and $t-2$ is given by:

$$
\begin{aligned}
p\left(\mathbf{S}_{2}(t) \mid \mathbf{S}_{2}(t-1), \mathbf{S}_{2}(t-2), \theta_{2}\right) & =p\left(\mathbf{S}_{2}(t)-\phi_{1} \mathbf{S}_{2}(t-1)-\phi_{2} \mathbf{S}_{2}(t-2) \mid \theta_{2}\right) \\
& =\mathcal{N}\left(\phi_{1} \mathbf{S}_{2}(t-1)+\phi_{2} \mathbf{S}_{2}(t-2), \sigma_{2}^{2}\right)
\end{aligned}
$$

Using the chain rule, we decompose the prior for $\mathbf{S}_{2}$ :

$$
\begin{aligned}
p\left(\mathbf{S}_{2}, \theta_{2}\right)= & p\left(\mathbf{S}_{2}(1) \ldots \mathbf{S}_{2}(T), \theta_{2}\right) \\
= & \left(\prod_{k=0}^{T-2} p\left(\mathbf{S}_{2}(T-k) \mid \mathbf{S}_{2}(T-k-1), \mathbf{S}_{2}(T-k-2), \theta_{2}\right)\right) \\
& p\left(\mathbf{S}_{2}(2) \mid \mathbf{S}_{2}(1), \theta_{2}\right) p\left(\mathbf{S}_{2}(1) \mid \theta_{2}\right)
\end{aligned}
$$


where $\quad p\left(\mathbf{S}_{2}(2) \mid \mathbf{S}_{2}(1), \theta_{2}\right)=\mathcal{N}\left(\phi_{1} \mathbf{S}_{2}(1), \sigma_{2}^{2}\right), \quad p\left(\mathbf{S}_{2}(1) \mid \theta_{2}\right)=\mathcal{N}\left(0, \sigma_{2}^{2}\right), \quad \theta_{2}=$ $\left(\phi_{1}, \phi_{2}, \sigma_{2}^{2}\right)$.

$$
\begin{aligned}
p\left(\mathbf{S}_{2} \mid \theta_{2}\right) \propto \exp ( & -\frac{1}{2 \sigma_{2}^{2}}\left(\left\|\mathbf{S}_{2}(1)\right\|_{2}^{2}+\left\|\mathbf{S}_{2}(2)-\phi_{1} \mathbf{S}_{2}(1)\right\|_{2}^{2}\right. \\
& \left.\left.+\sum_{k=2}^{T}\left\|\mathbf{S}_{2}(k)-\phi_{1} \mathbf{S}_{2}(k-1)-\phi_{2} \mathbf{S}_{2}(k-2)\right\|_{2}^{2}\right)\right)
\end{aligned}
$$

With this prior, the precise structure of the frequencies of the trailing edge source $S_{2}$ is constrained but in the other hand, there are more parameters to fix or to learn ( $\phi_{1}, \phi_{2}$ and $\sigma_{2}$ while there is just $\alpha_{2}$ for the Laplace distribution).

\section{Algorithms}

In both case, we use a classical Maximum A Posteriori to look for the solutions $\widehat{\mathbf{S}}, \widehat{\mathbf{S}_{1}}$ and $\widehat{\mathbf{S}_{2}}$ by minimizing the following cost function:

$$
\begin{aligned}
J(\mathbf{A}, \mathbf{S}) & =-\log \left(p\left(\mathbf{X} \mid \mathbf{A}, \mathbf{S}_{1}, \mathbf{S}_{2}, \sigma_{E}^{2}\right) p\left(\mathbf{A}, \sigma_{A}^{2}\right) p\left(\mathbf{S}_{1}, \sigma_{1}^{2}\right) p\left(\mathbf{S}_{2}, \theta_{2}\right)\right) \\
& =\frac{1}{2 \sigma_{E}^{2}}\left\|\mathbf{X}-\mathbf{A}_{1} \mathbf{S}_{1}-\mathbf{A}_{2} \mathbf{S}_{2}\right\|_{2}^{2}+\frac{1}{2 \sigma_{A}^{2}}\|\mathbf{A}\|_{2}^{2}+\frac{1}{2 \sigma_{1}^{2}}\left\|\mathbf{S}_{1}\right\|_{2}^{2}+\Omega\left(\mathbf{S}_{2}, \theta_{2}\right)
\end{aligned}
$$

Depending on the precise choice of the prior probabilities $p\left(\mathbf{S}_{2}, \theta_{2}\right)$, the $\Omega\left(\mathbf{S}_{2}, \theta_{2}\right)$ term of the function $J$ will take different forms but the general scheme will be the same for all the algorithms. Since there is no closed-form for the global minimizer of the previous criterion, we will use an alternative minimization iterative procedure to estimate the solution. Each iteration is made of three steps:

- estimation of $\mathbf{A}$ with known $\left(\mathbf{S}_{1}, \mathbf{S}_{2}\right)$;

- estimation of $\mathbf{S}_{1}$ with known $\mathbf{A}$ and $\mathbf{S}_{2}$;

- estimation of $\mathbf{S}_{2}$ with known $\mathbf{A}$ and $\mathbf{S}_{1}$.

The first two steps can be solved in closed-form but this is not the case for the third step. We thus need some numerical optimization schemes depending on the prior chosen.

For the Laplace prior, the optimization problem can be solved by a LASSO algorithm [4] which can be written as:

$$
J\left(\mathbf{A}, \mathbf{S}_{1}, \mathbf{S}_{2}\right)=\frac{1}{2 \sigma_{E}^{2}}\left\|\mathbf{X}-\mathbf{A}_{1} \mathbf{S}_{1}-\mathbf{A}_{2} \mathbf{S}_{2}\right\|_{2}^{2}+\frac{1}{2 \sigma_{A}^{2}}\|\mathbf{A}\|_{2}^{2}+\frac{1}{2 \sigma_{1}^{2}}\left\|\mathbf{S}_{1}\right\|_{2}^{2}+\alpha_{2}\left\|\mathbf{S}_{2}\right\|_{1}
$$

Due to the temporal dependency for the $\operatorname{AR}(2)$ prior, we cannot get a global solution for the matrix $\mathbf{S}_{2}$ and need to do the estimation for each column of the 
TABLE 1. Computation time (mean on 10 runs)

\begin{tabular}{ll} 
Prior & Time \\
\hline Gaussian & $0.016 \mathrm{~s}$ \\
AR2 prior & $2.5 \mathrm{~s}$ \\
Laplace prior & $130 \mathrm{~s}$
\end{tabular}

matrix (representing the time). The cost function for the estimation of the source at time $t$ (and by removing terms which do not depend on $\mathbf{S}_{2}$ ) can be written as

$J\left(\mathbf{S}_{2}(t)\right)=\frac{1}{2 \sigma_{E}^{2}}\left\|\mathbf{X}(t)-\mathbf{A}_{1} \mathbf{S}_{1}(t)-\mathbf{A}_{2} \mathbf{S}_{2}(t)\right\|_{2}^{2}+\frac{1}{2 \sigma_{2}^{2}}\left\|\mathbf{S}_{2}(t)-\phi_{1} \mathbf{S}_{2}(t-1)-\phi_{2} \mathbf{S}_{2}(t-2)\right\|_{2}^{2}$

The minimizer of the previous criterion, for a fixed $t$, is known in closed-form since it is simply a Tikhonov regularization problem [5].

\section{EXPERIMENTAL RESULTS}

We present here experiments on simulated data. Three different algorithms are used: the first one, taken as a reference, uses a simple Gaussian prior model for the trailing edge source; the second one uses the Laplace prior and the last one uses the $\mathrm{AR}(2)$ prior (all results presented here are computed on a 4 cores Intel Core i5-3570 CPU with 8Gb memory running MATLAB r2013a, all the LASSO problems are solved using the built-in lasso MATLAB function).

Our simulation follows the experimental setup as described previously: two sources, a large band source on the airfoil and a narrow band source on the trailing edge, two sets of sensors, a first set which is longitudinal to the airflow and a second set on the trailing edge. The narrow band source is produced from a white noise filtered by an order 8 Butterworth filter centered between $2400 \mathrm{~Hz}$ and $3100 \mathrm{~Hz}$ and the signal for the large band source is directly taken from the signal received by the longitudinal microphone which is the farthest from the trailing edge (since it does not hear at all the narrow band source). We have a power ratio of $40 \mathrm{~dB}$ between the two sources, and an additive white noise of $20 \mathrm{~dB}$ is added. For these experiments, we generate 10 blocks of 2048 points. FIGURE 4 presents the spectra of two sensors (one from the longitudinal set and another one from the transverse set, see FIGURE 2 for exact sensors position), and we need to increase the power ration between sources to $0 \mathrm{~dB}$ in order to make the power peak visible on the spectrum from the transverse sensor (FIGURE 4, sensor T02).

We see on FIGURE 5 that the two algorithms with a sparsity constraint ( $5 \mathrm{c}$ and $5 \mathrm{~d})$ are able to recover the sources signal in a satisfactory way: both the position of the power peak (around $2800 \mathrm{~Hz}$ ) and the power ratio between large and narrow band sources (40dB) are recovered successfully. Not surprisingly, the Gaussian prior is completely inefficient, confirming the need for sparsity inducing priors for the 


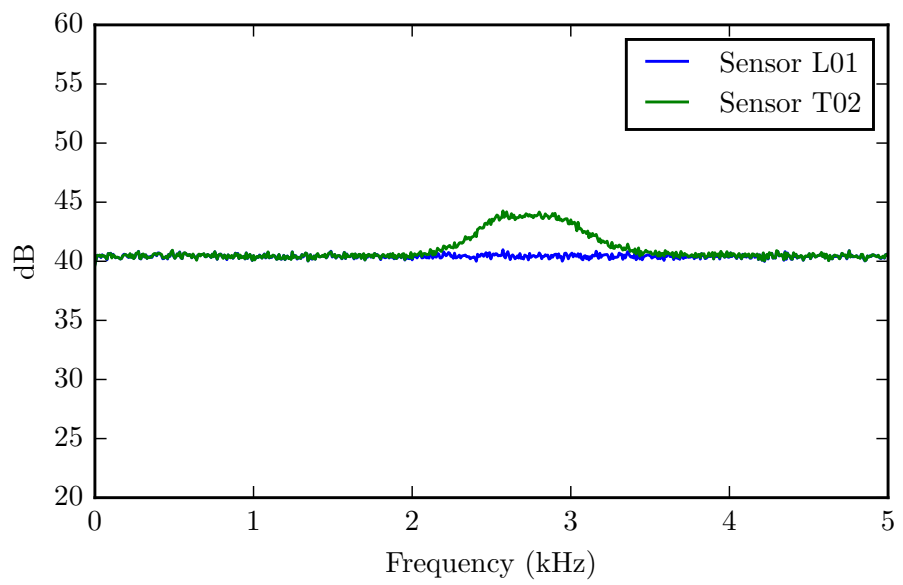

FIGURE 4. Spectrum of the mixture received by a longitudinal sensor (sensor L01) and a transverse sensor (sensor T02)

(a) Truth

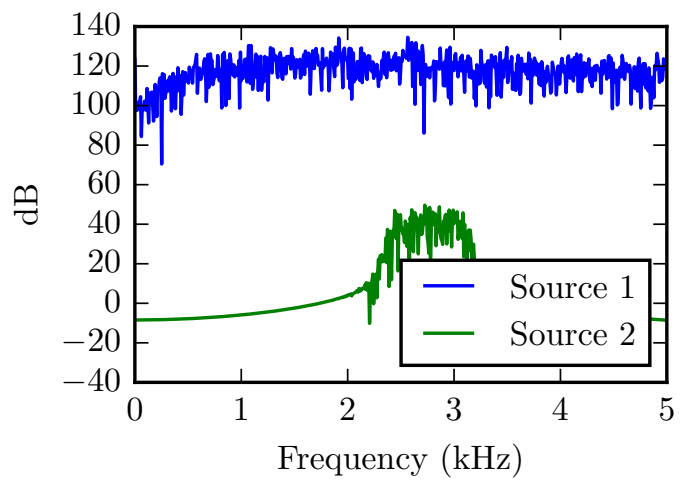

(c) Laplace prior

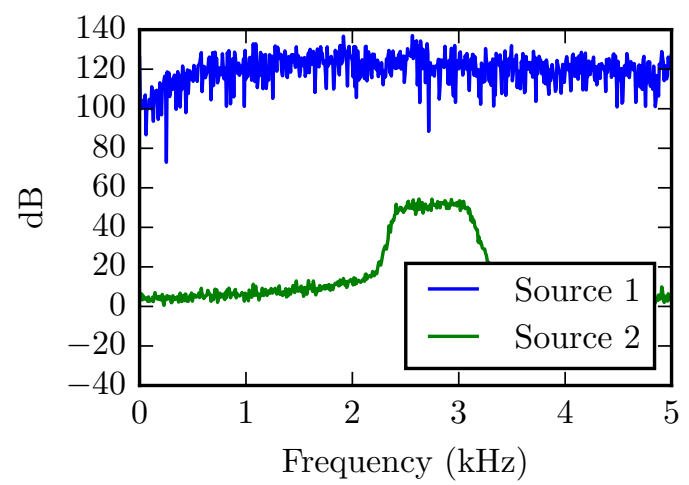

(b) Gaussian prior on both sources

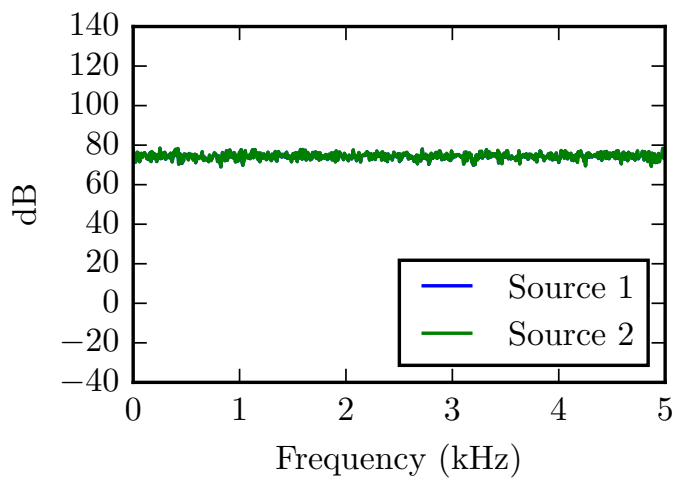

(d) $\operatorname{AR}(2)$ prior

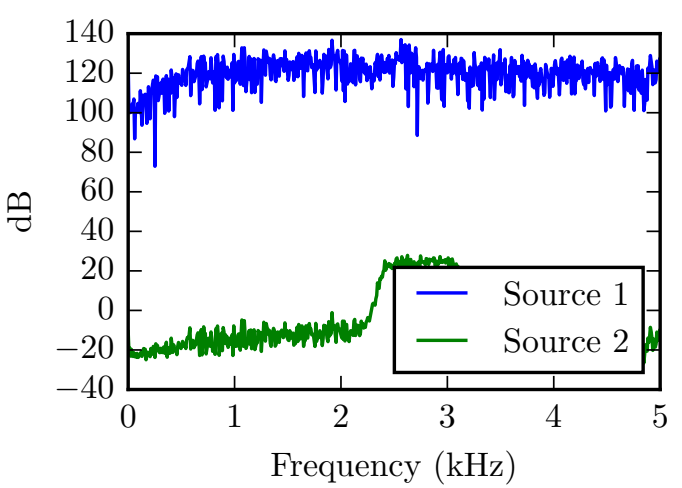

FIGURE 5. Truth and reconstructed spectra (large band source in blue, narrow band source in green, mean on 10 runs) 
trailing edge source. It is also interesting to notice that although the Laplace prior does not constraint the power distribution to be unimodal, we still retrieve a unique peak.

The main difference between the two sparsity constraining methods can be seen in TABLE 1. The Maximum A Posteriori problem with the Laplace prior is much more difficult than the one with the $\mathrm{AR}(2)$ prior since we need to solve a LASSO problem which is more computationally demanding than directly applying the formulas for the $\operatorname{AR}(2)$ prior.

\section{CONCLUSION}

We presented a preliminary study about time domain source separation for wind tunnel observations. The first method, using a Laplace prior, works in the frequency domain and imposes only the sparsity of the spectrum. The second method, using an auto-regressive model, works in the time domain but puts more constraints on the shape of the spectrum (not only sparse, but also unimodal). Both methods are efficient on the simulated data but the algorithm using the auto-regressive prior is much faster than the one using the Laplace prior.

More work is needed to apply these methods on the real dataset: we will have to improve our simulation by using more trailing edge sources (instead of a unique one) and by taking into account in our Bayesian approach the decorrelation which will appear between the signals heard by the sensors.

\section{REFERENCES}

1. N. Chu, A. Mohammad-Djafari, J. Picheral, and N. Gac, "An invariant convolution model and its Variational Bayesian Approximation approach via Students-t priors for acoustic imaging in colored noises," in Proceedings of the 21st International Congress on Acoustics, Montreal, Canada, 2013, vol. 19, p. 055031 (9pages).

2. N. Chu, J. Picheral, A. Mohammad-Djafari, and N. Gac, Applied Acoustics 76, 197-208 (2014).

3. J. Mairal, Sparse coding for machine learning, image processing and computer vision, These, École normale supérieure de Cachan - ENS Cachan (2010).

4. R. Tibshirani, Journal of the Royal Statistical Society. Series B (Methodological) pp. 267-288 (1996).

5. A. N. Tikhonov, Numerical methods for the solution of ill-posed problems, vol. 328, Springer, 1995. 